\title{
Immunity and early atherosclerosis in the course of systemic lupus erythematosus, mixed connective tissue disease and antiphospholipid syndrome
}

\author{
Ewa Haładyj ${ }^{1}$, Agnieszka Paradowska-Gorycka ${ }^{2}$, Anna Felis-Giemza ${ }^{1}$, Marzena Olesińska $^{1}$ \\ ${ }^{1}$ Department of Connective Tissue Diseases, National Institute of Geriatrics, Rheumatology and Rehabilitation, Warsaw, Poland \\ ${ }^{2}$ Department of Biochemistry and Molecular Biology, National Institute of Geriatrics, Rheumatology and Rehabilitation, Warsaw, Poland
}

\begin{abstract}
Atherosclerosis is a chronic inflammatory disease of the arteries associated with various risk factors that promote lipid abnormalities, development and progression of atherosclerotic lesions, plaque rupture, and vascular thrombosis. Atherosclerosis is accelerated in autoimmune diseases. Non-invasive investigations showed increased intima-media thickness (IMT), carotid plaque, and coronary artery calcifications in patients with antiphospholipid syndrome, systemic lupus erythematosus and mixed connective tissue disease compared to controls. The balance between the proinflammatory and anti-inflammatory cytokines allows the immune equilibrium to be maintained. In autoimmune diseases the prevalence of proinflammatory factors leads to premature atherosclerosis. This review presents complementary knowledge on innate and adaptive immunity, cytokines and the role of inflammasomes in progression of early atherosclerosis.
\end{abstract}

Key words: atherosclerosis, interleukins, transforming growth factor $\beta$, interferon $\gamma$, tumor necrosis factor $\alpha$, adipokines, peroxisome proliferator-activated receptors, toll-like receptors.

\section{Introduction}

Development of atherosclerosis (AT) and cardiovascular disease (CVD) in systemic lupus erythematosus (SLE), mixed connective tissue disease (MCTD) and antiphospholipid syndrome (APS) involves both acquired, modifiable risk factors (e.g., hypercholesterolemia, tobacco smoking) and genetic factors. Autoantibodies, autoantigens and autoreactive lymphocytes are also involved in these processes. Additionally, oxidative stress together with dyslipidemia and various systemic inflammation mediators, including cytokines, chemokines, prothrombotic molecules and adhesion receptors, has been implicated in the development of these vascular pathologies [1, 2].

Systemic lupus erythematosus is a chronic, inflammatory disease characterized by a perturbed immune response with the consequent production of autoantibodies. Some of these autoantibodies form immune complexes that are responsible for tissue inflammation directly contributing to the development of different clinical manifestations of the disease. Clinically SLE is characterized by a plethora of disease manifestations, including nephritis, arthritis, pleuritis, pericarditis and vasculitis. A typical serological feature of SLE is the protean production of autoantibodies of different specificities and, possibly related to this, disturbed apoptosis [3]. It has been suggested that the local inflammatory and autoimmune processes can promote AT in SLE patients [4]. Patients with SLE have a higher risk of CVD than in the general population, and it occurs in younger individuals. Systemic lupus erythematosus itself has been shown to be an independent risk factor of AT progression. Relevant factors directly influencing the development of AT and CVD in SLE comprise drug therapy, immune complex generation and changes in innate immune responses, complement activation, endothelial dysfunction, oxida-

Address for correspondence:

Ewa Haładyj, Department of Connective Tissue Diseases, National Institute of Geriatrics, Rheumatology and Rehabilitation, Spartanska 1, 02-637 Warsaw, Poland, e-mail: ehaladyj@02.pl

Submitted: 12.04.2016; Accepted: 18.08.2016 
tive stress, increased production of adipokines, dysfunctional lipids and changes in the production and activity of a complex network of cytokines [5].

Antiphospholipid syndrome is a disease of unknown etiology, characterized by the presence of antiphospholipid antibodies (aPL) directed against the proteins forming complexes with phospholipids of cell membranes and coexisting thrombotic events or pregnancy losses. The ongoing immune process alters the endothelium of blood vessels, platelets and coagulation proteins. It leads to the development of arterial and/or venous thrombosis. Endothelial dysfunction is in the meantime a basis for premature AT.

Mixed connective tissue disease (MCTD) is a chronic systemic inflammatory disease characterized by clinical feature of puffy hands with Raynaud phenomenon in the presence of anti-U1RNP antibodies (anti-U1RNP) [6]. The constellation of other clinical symptoms specific for SLE, systemic sclerosis and inflammatory myositis is variable in each patient. A common finding in all patients is chronic inflammation with associated vasculopathy affecting both small and large vessels. The vascular lesions frequently lead to pulmonary hypertension. Endothelial dysfunction is an early marker of both vasculopathy and premature AT. In MCTD coexisting with CVD compared with MCTD itself, increased titers of anti-U1RNP, IgG and IgM anticardiolipin antibodies ( $\mathrm{aCL}$ ) and antiendothelial antibodies (AECA) have been demonstrated. It may prove that presence of aPL or AECA with anti-U1RNP in MCTD patients may exacerbate endothelial dysfunction by increasing proinflammatory and procoagulative effects.

\section{Adaptive immunity in atherosclerosis}

The importance of the adaptive immune response in AT has gradually risen. It is widely acknowledged that atherosclerosis is caused by an imbalance between pathogenic T cells and Tregs, which suppress the overactive immune response to autoantigens. In the atherosclerotic inflammatory process the role of Tregs is also important. The direct role of Tregs in experimental atherosclerosis models initially reported depletion of peripheral Tregs, increased atherosclerotic lesion size and its vulnerability in ApoE-/-mice [7]. In contrast, adoptive transfer of Tregs can greatly reduce plaque size [7]. Additionally, generation of HSP60-specificTregs could inhibit the formation of plaques in murine AT [8]. Similarly, induction of oral tolerance with antigen-specific Tregs could attenuate experimental AT. Furthermore, some researchers have found a reduced number of naturally occurring Tregs with impaired suppressive properties in patients with acute coronary syndrome who have coronary AT [9].
The number of Tregs in patients with acute coronary syndrome was elevated and the inhibitory effects on the effectors $T$ cell proliferation were enhanced after shortterm treatment with atorvastatin [9]. The inhibitory effects of atorvastatin on inflammation in acute coronary syndrome may be caused by its effects on Tregs and consecutive restoration of immune homeostasis. Only a few reports focus on the peripheral blood Tregs - one of them addressed the presence and localization of Tregs in human atherosclerotic plaque tissues. It showed that Tregs were present in a low amount at plaques in all developmental stages of human plaque formation within the intima and adventitia. This may explain the smoldering chronic inflammatory process that takes place throughout the long standing course of AT [10].

\section{Cytokines in atherosclerosis}

Atherosclerosis, like other chronic autoimmune diseases such as SLE, APS, and MCTD, can be characterized by activation of macrophages and lymphocytes, alteration in the Th1/Th2 ratio, and elevation of inflammatory cytokines. A number of growth factors and cytokines including platelet-derived growth factor (PDGF), tumor necrosis factor $\alpha$ (TNF- $\alpha$ ), interleukin 1 (IL-1), monocyte chemotactic protein 1 (MCP-1, CCL2), interferon $\gamma($ IFN- $\gamma$ ), and macrophage colony-stimulating factor (M-CSF) have been identified in human atherosclerotic plaques, mainly obtained from carotid endarterectomy specimens. An important source of cytokines in the atherosclerotic plaque is macrophages. Macrophages produce both proinflammatory cytokines - TNF- $\alpha$, IL-1, IL-6, IL-12, IL-15, and IL-18 - and the anti-inflammatory cytokines IL-10 and transforming growth factor $\beta$ (TGF- $\beta$ ). Many cytokines are expressed highly in atherosclerotic regions and exhibit pro- and antiatherogenic actions. An important outcome derived from the studies reporting on this area is that only for a few cytokines is there sufficient data allowing them to be classified as typically proatherogenic (IL-6, IL-17, IFN- $\gamma$, TNF- $\alpha$, BAFF, MIF, etc.) or antiatherogenic (IL-10), and that some cytokines (IFN- $\gamma$, TNF- $\alpha$, IL-4, $\mathrm{IL}-6)$ can exert pro- or antiatherogenic effects depending on the disease status.

\section{Proatherogenic cytokines}

Interleukin $1 \beta$ is considered to be a potent pro-inflammatory cytokine with potential deleterious effects if produced uncontrollably. Release of IL-1 may precede caspase-1-induced inflammatory cell death in response to infections called pyroptosis, with both apoptosis and necrosis [11]. Production of pro-IL-1 $\beta$ and nucleotide-binding domain rich protein 3 (NLRP3) activation can also be mediated through cytokine trans-membrane 
receptors (TNF receptor) or pattern recognition receptors such as toll-like receptors (TLRs). Released pro-inflammatory IL-1 $\beta$ cytokine can then activate other cells or self-perpetuate this activation cycle by binding the IL-1R in a feed-forward loop [12]. Interleukin $1 \beta$ is a pro-inflammatory cytokine, and its synthesis and release are tightly regulated. Interleukin $1 \beta$ activity is further controlled by IL-1 receptor antagonist (IL-1Ra) [12]. Important evidence for the role of IL-1 $\beta$ in auto-inflammatory diseases came from clinical studies showing IL-1 $\beta$ blockade as a very efficient treatment. Endotoxin stimulation increased IL-1 $\beta$ more than IL-1Ra (it promoted a proinflammatory state). A polymorphism of IL-1Ra and increased IL-1Ra were associated with decreased mean coronary artery plaque. Activation of innate immunity changed the balance between IL-1 $\beta$ and IL-1Ra in atherosclerotic arteries and initiated the proinflammatory state. Thus, the presence of an IL-1Ra intron 2 polymorphism increasing IL-1Ra, and possibly the IL-1Ra: IL-1 $\beta$ ratio, were associated with reduced coronary AT.

Activated caspase-1 controls maturation of the IL-1 family of cytokines. Multi-molecular complexes of nucleotide-binding domain leucine-rich repeat receptors (NLRs) regulate inflammatory caspases and IL-1 [13]. Nucleotide-binding domain leucine-rich repeat receptors is a family of intracellular (cytoplasmic) immune receptors with more than 20 members in humans. NLRP1, NLRP3 and NLRC4 can activate pro-inflammatory caspases in response to various endogenous stimuli. Pro-caspase-1/ NLRP3 inflammasome complexes activate caspase-1 to proteolytically cleave pro-IL-1 $\beta$ (35 kDa) into the bioactive cytokine IL-1 $(17 \mathrm{kDa})$ [14]. Nucleotide-binding domain leucine-rich repeat receptors are thought to act as danger sensors to exogenous, but mostly to endogenous signals, generated by either infectious or cellular (metabolic) stress. The substances able to activate the NLRP3 inflammasome are diverse in nature and structure: they include infectious agents (Leishmania spp., Staphylococcus spp., Listeria spp., influenza A virus) and toxins (LPS), particles (microcrystals in gout and pseudogout) and metabolic alterations (low potassium, ATP, hyperglycemia, oxidative stress) [13].

Another proinflammatory interleukin, IL-6, is involved in the recruitment of inflammatory cells and lipid homeostasis. Interleukin 6 is associated with increased cardiovascular mortality and prognosis in the general population [15]. Interleukin 6 stimulates hepatocytes to produce C-reactive protein (CRP) and other markers of inflammation [16]. C-reactive protein is not only a marker of the acute phase reaction but may also have a direct effect on leukocyte recruitment and apoptosis in the vessel wall [17]. Interleukin 6 is an independent marker of increased mortality in CVD through CRP pro- duction. Interleukin-6 is also required for short-term regulation of paraoxonase, an antioxidant enzyme present in high-density lipoproteins (HDL) [18]. In patients with unstable coronary syndrome, both IL-6 and CRP are elevated. In addition, IL-6 has been described as an independent predictor of endothelial dysfunction in rheumatoid arthritis [19]. Its role in AT progression in SLE is unclear; high IL-6 concentrations are linked to atherosclerotic risk in certain cohorts [20], but not in other cross sectional and longitudinal studies [21]. Interleukin 6 promotes and at the same time antibodies against IL- 6 or the IL- 6 receptor inhibit immunoglobulin and anti-dsDNA antibody production in vitro by $B$ cells in SLE. Circulating factors such as TNF- $\alpha$, IL- 6 and antibodies to lipoprotein lipase (anti-LPL) control some of the lipid alterations seen in SLE by reduced activity of the enzyme (LPL). LPL is responsible for the catabolism of chylomicrons and very-low-density lipoproteins (VLDL). Suppression of LPL activity results in accumulation of TG-rich particles. Tumor necrosis factor $\alpha$ and IL- 6 also trigger hepatic production of CRP, which may further exacerbate HDL suppression. At the same time, HDL have been shown to inversely correlate with high circulating IL-6 concentrations [22].

Expansion of the Th17 population and elevation of serum IL-17 have been clearly demonstrated in patients with SLE [19]. Interleukin 17 is believed to promote disease activity in SLE - high IL-17 and IL-23 have been reported in human SLE sera [20]. Interleukin 17 could stabilize the atherosclerotic plaque partly by suppressing the Th1 cell activity via IFN- $\gamma$ and promoting the Th2 cell activity via IL-5 [14]. T cells which produce both IL-17 and IFN- $\gamma$ were demonstrated to reside in the specimens of atherosclerotic plaque from patients with coronary heart disease [23]. Furthermore, in patients with acute coronary syndrome, a higher number of circulating Th17 cells and IL-17, as well as its related cytokines such as IL-6 and IL-23, were demonstrated as compared with those with stable angina and healthy individuals [24]. Interleukin 17 has been implicated in the development of atherosclerotic plaques in non-lupus models. Indeed, depleting IL-17R by knocking out the IL-17R gene of LDL receptor deficient atherosclerosis-prone mice reduced the size of aortic atherosclerotic plaques in those mice fed with a Western-type diet [25].

\section{Antiatherogenic cytokines}

Interleukin-10 is a Th2 cytokine with inhibitory effects on pro-inflammatory Th1 cells, endothelial cells, granulocytes and monocytes/macrophages. However, IL-10 can also be an immune stimulator by promoting antibody production and B-cell activation [26]. Interleukin-10 is produced in atherosclerotic lesions and 
correlates with diminished expression of inflammatory mediators. Increased IL-10 in serum is associated with improved systemic endothelial vasoreactivity in patients with elevated CRP in serum, demonstrating that the pro- and anti-inflammatory balance is a major determinant of the endothelial function. It has been reported that concentrations of IL-10 in patients with APS are reduced. Several authors have reported that IL-10 is raised in SLE patients, and it has been hypothesized as being one of the causative factors in SLE by induction of autoantibodies (e.g. against dsDNA) and of apoptosis [27]. In the light of this, it is surprising that findings from animal models indicate that IL-10 is an antiatherogenic cytokine [27].

Transforming growth factor $\beta$ likely plays a protective role against atherosclerotic plaque formation, as discussed in several publications [28]. Thus, the balance of proinflammatory and anti-inflammatory cytokines and their interactions with inflammatory cells and lipid components contributes to the formation and maintenance of the atherosclerotic plaque. Transforming growth factor $\beta$ deletion, specifically in T cells, accelerates AT. A study by Gojova et al. [29] examined the efficiency of TGF- $\beta$ activation in SLE patients with early AT. They found an inverse correlation between TGF- $\beta$ activation and LDL along with IMT values, where SLE patients with higher IMT and LDL had decreased TGF- $\beta$ activation. These trends were not found in control patients, suggesting that this phenomenon was specific to SLE with CVD.

\section{Interleukins with pro- or antiatherogenic effects depending on the disease status}

Interferon $\alpha$ is involved in atherosclerosis through several different mechanisms. It was found that low-density lipoprotein (LDL) receptor-deficient mice after receiving an injection of IFN- $\alpha$ had significantly accelerated atherosclerosis accompanied by increased plasma cholesterol and triglycerides. On the other hand, IFN- $\alpha$, mainly produced by the plasmacytoid dendritic cells (pDCs), is crucial in the pathogenesis of SLE, and IFN- $\alpha$ is increased in the majority of patients with SLE. In SLE, IFN- $\alpha$ promotes endothelial progenitor cell deletion and endothelial dysfunction, and causes abnormal vascular repair [30]. Plasmacytoid dendritic cells produce IFN- $\alpha$ combined with lipopolysaccharide in atherosclerotic plaque. It leads to an increase of toll-like receptor 4 (TLR4) expression and enhanced TNF- $\alpha$, IL-12 and matrix metalloproteinase 9 (MMP-9) production. All these processes can result in loss of atherosclerotic plaque stability [31]. Plaque disruption in atherosclerosis can be a trigger due to enhanced cytotoxic $T$ cell activities by IFN- $\alpha$ [32]. pDCs residing in atherosclerotic plaques pro- duce IFN- $\alpha$, which locally induces adjacent CD4+ T cells to express TNF-related apoptosis inducing ligand (TRAIL) [33]. In the context of chronic inflammation, TRAIL was demonstrated to prevent atherosclerotic plaque formation. Meanwhile, TRAIL deficiency was shown to be associated with calcification in atherosclerosis in a mouse model [33]. TRAIL potentially causes plaque rupture and acute coronary events. Interferon $\alpha$ induces myeloid dendritic cells in atherosclerotic plaques to produce inflammatory cytokines and matrix metalloproteinases. It can lead to destabilization of plaques [34]. On diseased endothelium in a P-selectin-dependent fashion, IFN- $\alpha$ also induces platelet aggregation and thrombosis. Platelets in SLE have been shown to have heightened IFN signatures. In this way platelets can activate plasmacytoid dendritic cell and subsequent IFN- $\alpha$ production through the interaction between CD40 and CD40L, potentially perpetuating endothelial toxicity and vascular thrombosis by further activating platelet aggregation as a positive feedback loop [35].

Interferon $\gamma$ expression is significantly increased in mononuclear cells of peripheral blood of SLE patients. The progression of AT has been well debated due to evidence conveying both pro- and antiatherogenic actions of IFN- $\gamma$. Interferon $\gamma$ is known as a proinflammatory cytokine, but it can also display anti-inflammatory properties. It is likely that IFN- $\gamma$ acts in both ways in AT. Interferon $\gamma$ has also been detected in atherogenic plaques [36]. It is a powerful growth inhibitor for smooth muscle cells, endothelial cells and collagen production, and thus promotes plaque instability. Interferon $\gamma$ in the atherosclerotic plaque can be produced by CD4+ Th1 cells, CD8+ $T$ cells and NKT cells. Macrophages have also been reported to produce IFN- $\gamma$. After all, IFN- $\gamma$ per se promotes oxidative stress and further endothelial damage [14]. Recent studies have confirmed and extended the role of IFN- $\gamma$ in lipid metabolism. Through up-regulation of the scavenger receptor for phosphatidyl serine and oxLDL, IFN- $\gamma$ induced foam cell formation. It is possible that its proatherogenic actions out-weight its antiatherogenic ones. Interferon $\alpha$ inhibits apoE and the ATP-binding cassette transporter $\mathrm{A} 1$ (ABCA1), resulting in decreased cholesterol efflux from macrophages. Interferon $\gamma$ modulates the ability of monocytes/macrophages to oxidize LDL. Interferon $\gamma$ inhibits the macrophage-induced oxidation of LDL, whereas TNF- $\alpha$, IL-4 and IL-13 enhance the ability of cell-mediated oxidation. Interferon $\gamma$ causes increased synthesis of TNF- $\alpha$ and IL-1 and improves the efficiency of antigen presentation. The administration of IFN- $\gamma$ accelerates atherosclerosis in apoE knockout mice. Additionally, increased serum IFN- $\gamma$ and IL-12 concentrations have been found in humans with unstable and stable angina compared with controls [37]. 
Tumor necrosis factor $\alpha$ and IL-1 are also present in human atherosclerotic lesions. Similarly to IFN- $\gamma$, they also affect smooth muscle proliferation. By stimulating the activation of macrophages, TNF- $\alpha$ and IL-1 induce local inflammation in blood vessels and secretion of matrix metalloproteinases, and promote expression of cell surface adhesion molecules [36]. Also TNF- $\alpha$ and IL-1 enhance production of M-CSF, GM-CSF, and G-CSF by monocytes, smooth muscle cells and endothelial cells. They activate and stimulate monocytes to transform into macrophages and foam cells. In apoE knockout mice inhibition of TNF- $\alpha$ decreased the progression of atherosclerosis. Elevated TNF- $\alpha$ may also play a role in the increased risk of atherosclerosis in the general population.

Tumor necrosis factor $\alpha$ has been identified in human endothelial and smooth muscle cells in all stages of atherosclerosis, from early intima thickening to established occlusive atherosclerosis. Tumor necrosis factor $\alpha$ has been associated with an elevated risk of recurrent myocardial infarctions and cardiovascular death after a first myocardial infarct. Tumor necrosis factor $\alpha$ induces adhesion molecule expression and enhances the recruitment of $T$ cells and monocytes to the endothelial cells. Elevated circulating TNF- $\alpha$ in SLE patients has been found to be associated with high triglyceride and low HDL. Tumor necrosis factor $\alpha$ concentrations are correlated with the burden of AT as assessed by carotid ultrasound among healthy middle-aged men. Tumor necrosis factor $\alpha$ elevation was shown to be associated with higher coronary calcium scores in patients with SLE [38].

The cytokine tumor necrosis factor (TNF)-like weak inducer of apoptosis (TWEAK, TNFSF12) is a member of the TNF superfamily that is prominently featured in normal and pathological remodeling of tissues [39]. TWEAK is primarily expressed as a soluble cytokine by infiltrating leukocytes. TWEAK is involved in pro-inflammatory responses, vascular activation and angiogenesis, cell growth, cell death, fibrogenic responses, and progenitor responses.

The presence of soluble TWEAK (STWEAK) is linked to increased rates of atherosclerosis, inflammation, angiogenesis, and apoptosis. The combination of high plasma STWEAK and high IL-6 was associated with increased cardiovascular-related mortality and all-cause mortality in patients undergoing hemodialysis [40]. High plasma TWEAK was observed in SLE patients with atherosclerosis. High urinary TWEAK was significantly associated with lupus nephritis, so it might be its potential biomarker. Administration of TWEAK to apolipoprotein E-deficient mice resulted in a significant increase in plaque, which was inhibited when mice were pre-treated with anti-TWEAK antibodies. In contrast, other clinical data have suggested that plasma STWEAK is inversely related to subclinical atherosclerosis in the general population [40]. sTWEAK was significantly elevated in patients with acute myocardial infarct compared to patients with stable coronary disease and healthy controls. Higher STWEAK levels were associated with adverse short-term post-myocardial infarct outcomes. STWEAK also positively correlated with coronary artery calcification and increased mortality in patients undergoing hemodialysis [41].

\section{Adipokines/adipose-derived hormones}

Adipokines, produced by white adipose tissue, regulate metabolism and energy homeostasis. Leptin is an anorectic peptide that functions as a hypothalamic modulator of food intake, body weight and fat stores. Obese patients develop resistance to leptin similar to insulin resistance in type 2 diabetes, and high serum leptin levels are observed in overweight individuals. Hyperleptinemia in the general population is also associated with hypertension, metabolic syndrome and atherosclerosis [42], and leptin signaling also contributes to atherosclerosis progression [43]. In addition, leptin has been linked to increased oxidative stress and endothelial dysfunction [46]. Elevated leptin induces oxidative stress in endothelial cells and cardiomyocytes [44]. Elevated leptin has been observed in adult and pediatric SLE [44]. In addition, serum leptin is higher in SLE patients with carotid plaque compared to those without. Serum leptin is correlated with oxLDL in postmenopausal women, and decreased leptin after weight loss is predictive of reduced oxLDL. Leptin was found to be independently associated with carotid plaque and positively correlated with piHDL and oxidized phospholipids in SLE [45]. When exogenous leptin was administered to lupus-prone mice, formation of piHDL and atherosclerotic plaque was accelerated [46].

In contrast, high serum adiponectin is associated with low adipose tissue and low risk of atherosclerosis and metabolic syndrome. Adiponectin is lower in patients with CVD. Data on the link between adiponectin and atherosclerosis are limited and contradictory: in one study, adiponectin was associated with carotid plaque, but no correlation was found between adiponectin or leptin and coronary calcification in another SLE cohort. Mean leptin concentrations were significantly higher in the 40 patients with plaque than in those without plaque, and also weakly correlated with carotid IMT. In another cohort, adiponectin was significantly and independently associated with carotid plaque in SLE [47]. However, Chung et al. [41] found no significant relationship between leptin or adiponectin and coronary calcification measured by electron beam computed tomography in 109 SLE patients and 78 control subjects. 
Elevated adiponectin in peripheral blood is reported to promote a wide range of benefits at the site of blood vessel injury, such as the downregulation of adhesion molecules by attenuating the nuclear factor $\kappa \mathrm{B}$ pathway (NFKB) [48]. In the sub-endothelium of injured human arteries accumulated adiponectin inhibits monocyte adhesion to endothelial cells and ultimately inhibits the migration and proliferation of vascular smooth muscle, which prevents atherosclerotic plaque formation. In addition, adiponectin was found in blood vessel walls after experimental endothelial injury, and was strongly expressed around infarcted but not normal myocardium, supporting a role in vascular and endothelial remodeling. It has been suggested that hypoadiponectinemia is an independent risk factor for hypertension, and has a detrimental effect on aortic stiffness.

Induction of adiponectin is a major mechanism underlying the immunomodulatory effects of PPAR $\gamma$ agonists. The peroxisome proliferator-activated receptors (PPARs) are a family of transcription factors that regulate energy balance by promoting either energy deposition or energy dissipation. Under normal physiological conditions, PPAR $\gamma$ is mainly expressed in adipose tissue and regulates diverse functions such as the development of fat cells and their capacity to store lipids [49]. There are a number of possible explanations for the lack of efficacy of rosiglitazone in the presence of antinuclear antibodies and renal disease when administered after disease onset, despite effective induction of adiponectin expression. It might be that severe active disease simply overwhelms the protective effects of PPAR $\gamma$ agonists and increased adiponectin. Alternatively, it might be that more advanced disease specifically impairs adiponectin's protective effects by, for example, down-regulating adiponectin receptor expression. This latter possibility is suggested by work from other groups showing that inflammatory stimuli are able to down-regulate expression of adiponectin receptors [50].

Recent studies have evaluated the effects of PPAR $\gamma$ agonists on SLE due to their anti-inflammatory and immunomodulatory properties [50]. The PPAR $\gamma$ agonist rosiglitazone reduced disease progression in two different mouse models of lupus and lupus-associated AT. Macrophage-specific deletion of PPAR $\gamma$ leads to antinuclear antibody production and glomerulonephritis. More recently, a small cohort of human SLE patients received four-week pioglitazone treatment in a randomized, double-blind clinical trial. The results showed that pioglitazone reduced CRP, increased HDL cholesterol, and improved insulin sensitivity, suggesting a beneficial effect on markers of CVD associated with SLE. Another study involving lupus patients receiving pioglitazone demonstrated a reduction in CRP and an increase in HDL cholesterol after four weeks of treatment, suggesting a beneficial effect on CVD associated with SLE.

Resistin, another adipokine, mediates obesity-induced insulin resistance in a mouse model of obesity. Administration of anti-resistin antibodies improved blood glucose and insulin action. In humans, resistin has been associated with obesity, diabetes and inflammation in some studies [50]. The role of resistin in SLE is poorly defined. Concentrations of resistin were not higher than in controls in one study with SLE patients, although there were differences in leptin, adiponectin and visfatin [51]. A relatively weak association has been found between resistin and erythrocyte sedimentation rate (ESR), but no association with CRP, IL-6, or TNF- $\alpha$ was observed [50,51]. In another study [52], resistin was independently elevated in patients with SLE compared to age- and race-matched controls. Resistin also correlated with measures of disease severity, inflammation, renal dysfunction, and markers of renal dysfunction.

The role of the recently discovered adipocytokine visfatin was also investigated in SLE. Visfatin binds to the insulin receptor and is potentially secreted to compensate for insulin resistance. It is also pro-inflammatory and associated with unstable atherosclerotic lesions. Visfatin concentrations were significantly higher in patients with SLE than controls but were not associated with metabolic or inflammatory measures [53] in some recent population studies, including the Framingham Offspring Cohort [54]. Visfatin, previously known as pre-B-cell colony enhancing factor, activates human leukocytes and stimulates the production of cytokines such as IL-6. Elevated concentrations of visfatin were observed in SLE and may reflect inflammatory processes in that disease.

\section{Innate immunity in atherosclerosis}

The innate immune system is also involved in both the pathogenesis of autoimmune diseases and related atherosclerosis. In fact, adaptive immunity and innate immunity, as two indivisible parts, have a mutual influence on each other. A wealth of evidence indicates that Tregs exert a suppressive effect on the innate immune system. Suppressive activity of Tregs on atherosclerosis is mediated through affecting macrophages foam cell formation. Tregs can also inhibit the pro-inflammatory properties of oxLDL-induced macrophages by decreasing NO production and iNOS expression and steer macrophage differentiation toward an anti-inflammatory cytokine producing phenotype. Moreover, at the transcriptional level, macrophages are modulated by Tregs by clearly decreased NF-KB up-regulation and TLR2 expression. A decreased proinflammatory cytokine response to oxLDL of Treg-modulated macrophages has been ob- 
served. In addition, the influence of Tregs also extends to dendritic cells and B cells. In this way, Tregs regulate subsequent innate and adaptive immune responses.

Effective defense against pathogens invading the host is possible due to early innate immunity. Pathogen recognition receptors (PRRs) can recognize a broad range of pathogens and recognize dangerous non-self-molecules from self-molecules [54]. Toll-like receptors (TLRs) are one of the main families of PRRs. The TLR family consists of at least 11 members, among which TLR4 was the first identified receptor and was subsequently well studied and well characterized. TLR4 is a type I transmembrane glycoprotein comprising an extracellular, transmembrane and intracellular signaling domain, and is expressed mainly in the cells of the innate immune system such as macrophages, dendritic cells and epithelial cells (exogenous sensors). Ligand binding to TLR4 readily triggers homodimerization of TLR4, initiating the recruitment of its adaptor protein, myeloid differentiation factor 88 (MyD88; MYD88) [55].

Oxidation-specific epitopes present on apoptotic cells and their cellular debris may have provided the primary evolutionary selection of PRRs. Lipid peroxidation is ubiquitous and represents a major component of the inflammatory state associated with atherosclerosis. Thus, the generation of oxidation-specific epitopes may activate innate immunity through endogenous cytoplasmic sensors such as inflammasomes. TLR4, $\beta_{2}$ GP1 and annexin A2 form an activation cluster in plasma membrane microdomains of human monocytes after LPS or aPL ligation [56]. Whereas under resting conditions EC abundantly express TLR4 molecules, but sparsely TLR2 proteins, EC activation by TNF or TLR4 ligands increases the TLR2 and aPLA-associated response of EC. In humans, no TLR4 polymorphisms have been found to influence susceptibility to SLE. However, a polymorphism affecting the MyD88 adapter-like (MAL) protein used by TLR2 and TLR4 was associated with decreased susceptibility to SLE. This polymorphism may modify the intracellular signal generated by TLR2 and TLR4 activation. TLR2 and TLR4, in particular, have been shown to recognize fatty acids. Data in humans are scarce; however, genotypic studies have shown that the TLR2 R753Q single-nucleotide polymorphism is a risk factor for coronary restenosis. In addition, humans with a D299G polymorphism in the TLR4 gene, which impairs TLR4 signaling, show a decreased risk of AT $[57,58]$.

\section{Platelets}

Activated platelets play a well-recognized role in AT. Platelets are mediators of immunity and inflammation. Platelets, among other immunologically relevant molecules, release numerous cytokines and also ex- press CD40 ligand (CD40L/CD154), FcyRlla, and TLRs on their surface [59]. In SLE platelet activation is increased compared to controls [60]. It has been suggested that interplay between IFN- $\alpha$ and platelets causes vascular damage. The IFN- $\alpha$ signature has been described in the transcript of some platelets in SLE, extending to the protein level for selected IFN- $\alpha$-regulated genes. This type of IFN- $\alpha$ signature and platelet activation was prevalent in SLE patients with a history of vascular events, with upregulation of CD4OL on the surface of platelets in response to circulating immune complexes, and activation of plasmacytoid dendritic cells to release more IFN- $\alpha$ via a CD40L-CD40 interaction [60]. It has been reported that platelet depletion or inhibition with clopidogrel can improve nephritis and disease activity in murine models [60]. Furthermore, antiplatelet agents have a unique role in protecting against CVD in SLE in experimental models.

\section{Summary}

Atherosclerosis leads to cardiovascular disease and is accelerated in autoimmune diseases such as SLE, APS and MCTD. Risk factors of atherosclerosis in such patients consist of traditional risk factors and mechanisms specific to autoimmunity such as chronic inflammation, endothelial dysfunction, decreased vascular repair, effects of pro- and antiatherogenic cytokines, antibody formation, perturbed lipid homeostasis and the redox environment. The interplay between the innate and adaptive immune systems is decisive in continued research on mechanisms of atherosclerotic plaque formation which will hopefully provide effective tools and targets to improve survival and overall quality of life of patients with SLE, MCTD and APS.

The authors declare no conflict of interests.

\section{References}

1. Shoenfeld Y, Gerli R, Doria A, et al. Accelerated atherosclerosis in autoimmune rheumatic diseases. Circulation 2005; 112: 3337-3347.

2. Soltész P, Kerekes G, Dér H, et al. Comparative assessment of vascular function in autoimmune rheumatic diseases: considerations of prevention and treatment. Autoimmun Rev 2011; 10: 416-425.

3. Ruiz-Irastorza G, Khamashta MA, Castellino G, et al. Systemic lupus erythematosus. Lancet 2001; 357: 1027-1032.

4. Wick $G$, Schett A, Amberger A, et al. Is atherosclerosis an immunological mediated disease? Immunol Today 1995; 16: 27-33.

5. López-Pedrera C, Aguirre MA, Barbarroja N, et al. Accelerated atherosclerosis in systemic lupus erythematosus: role of proinflammatory cytokines and therapeutic approaches. J Biomed Biotechnol 2010; 2010: 607084. 
6. Szücs G, Tímár O, Szekanecz Z, et al. Endothelial dysfunction precedes atherosclerosis in systemic sclerosis-relevance for prevention of vascular complications. Rheumatology (Oxford) 2007; 46: 759-762

7. Ait-Oufella H, Salomon BL, Potteaux S, et al. Natural regulatory T cells control the development of atherosclerosis in mice. Nat Med 2006; 12: 178-180.

8. Yang K, Li D, Luo M, et al. Generation of HSP60-specific regulatory $\mathrm{T}$ cell and effect on atherosclerosis. Cell Immunol 2006; 243: 90-95.

9. Hu Z, Li D, Hu Y, et al. Changes of CD4+CD25+ regulatory T cells in patients with acute coronary syndrome and the effects of atorvastatin. J Huazhong Univ SciTechnol Med Sci 2007; 27: 524-527.

10. Tu H, Li Q Jiang $\mathrm{H}$, et al. Dual effects of statins therapy in systemic lupus erythematosus and SLE-related atherosclerosis: The potential role for regulatory T cells. Atherosclerosis 2012; 222: 29-33.

11. Stutz A, Golenbock DT, Latz E. Inflammasomes: too big to miss. J Clin Invest 2009; 119: 3502-3511.

12. Goldbach-Mansky R, Dailey NJ, Canna SW, et al. Neonatal-onset multisystem inflammatory disease responsive to interleukin-1B inhibition. N Engl J Med 2006; 355: 581-592.

13. Yang J, Chu Y, Yang X, et al. Th17 and natural Treg cell population dynamics in systemic lupus erythematosus, Arthritis Rheum 2009; 60: 1472-1483.

14. Madamanchi NR, Vendrov A, Runge MS. Oxidative stress and vascular disease. Arterioscler Thromb Vasc Biol 2005; 25: 29-38.

15. Stone NJ, Bilek S, Rosenbaum S. Recent National Cholesterol Education Program Adult Treatment Panel III Update: adjustments and options. Am J Cardiol 2005; 96: 53E-59E.

16. Chobanian AV, Bakris GL, Black HR, et al. The Seventh Report of the Joint National Committee on Prevention, Detection, Evaluation, and Treatment of High Blood Pressure: the JNC 7 report. JAMA 2003; 289: 2560-2572.

17. Costenbader KH, Karlson EW, Gall V, et al. Barriers to a trial of atherosclerosis prevention in systemic lupus erythematosus. Arthritis Rheum 2005; 53: 718-723.

18. Durán-Barragán S, McGwin G Jr, Vilá LM, et al. Angiotensin-converting enzyme inhibitors delay the occurrence of renal involvement and are associated with a decreased risk of disease activity in patients with systemic lupus erythematosus - results from LUMINA (LIX): a multiethnic US cohort. Rheumatology (Oxford) 2008; 47: 1093-1096.

19. Forrester JS, Libby P. The inflammation hypothesis and its potential relevance to statin therapy. Am J Cardiol 2007; 99 732-738.

20. Kajiwara T, Yasuda T, Matsuura E. Intracellular trafficking of beta2-glycoprotein I complexes with lipid vesicles in macrophages: implications on the development of antiphospholipid syndrome. J Autoimmun 2007; 29: 164-173.

21. Kuwana M, Matsuura E, Kobayashi K, et al. Binding of beta2-glycoprotein I to anionic phospholipids facilitates processing and presentation of a cryptic epitope that activates pathogenic autoreactive T cells. Blood 2005; 105: 1552-1557.

22. Toms TE, Panoulas VF, Kitas GD. Dyslipidaemia in rheumatological autoimmune diseases. Open Cardiovasc Med J 2011; 5: 64-75.
23. Medina-Gomez G, Gray S, Vidal-Puig A. Adipogenesis and lipotoxicity: role of peroxisome proliferator-activated receptor gamma (PPARgamma) and PPARgammacoactivator-1 (PGC1). Public Health Nutr 2007; 10: 1132-1137.

24. van Es T, van Puijvelde GHM, Ramos OH, et al. Attenuated atherosclerosis upon IL-17R signaling disruption in LDLr deficient mice. Biochem Biophys Res Commun 2009; 388: 261-265.

25. Chen S, Crother TR, Ardit M. Emerging role of IL-17 in atherosclerosis. J Innate Immun 2010; 2: 325-333.

26. Wade NS, Major AS. The problem of accelerated atherosclerosis in systemic lupus erythematosus: Insights into a complex co-morbidity. Thromb Haemost 2011; 106: 849-857.

27. Binder CJ, Hartvigsen K, Chang MK, et al. IL-5 links adaptive and natural immunity specific for epitopes of oxidized LDL and protects from atherosclerosis. J Clin Invest 2004; 114: 427437.

28. Bedi U, Singh M, Singh P, et al. Effects of statins on progression of coronary artery disease as measured by intravascular ultrasound. J Clin Hypertens (Greenwich) 2011; 13: 492-496.

29. Gojova A, Brun V, Esposito B, et al. Specific abrogation of transforming growth factor-beta signalling in $T$ cells alters atherosclerotic lesion size and composition in mice. Blood 2003; 102: 4052-4058.

30. Niessner A, Shin MS, Pryshchep O, et al. Synergistic proinflammatory effects of the antiviral cytokine interferon-alpha and Toll-like receptor 4 ligands in the atherosclerotic plaque. Circulation 2007; 116: 2043-2052.

31. Niessner A, Sato K, Chaikof EL, et al. Pathogen-sensing plasmacytoid dendritic cells stimulate cytotoxic T-cell function in the atherosclerotic plaquethrough interferon-alpha. Circulation 2006; 114: 2482-2489.

32. Levy Z, Rachmani R, Trestman S, et al. Low-dose interferon-gamma accelerates atherosclerosis in an LDL receptor-deficient mouse model. Eur J Intern Med 2003; 14: 479-483.

33. Secchiero P, Rimondi E, di lasio MG, et al. C-Reactive protein downregulates TRAIL expression in human peripheral monocytes via an Egr-1-dependent pathway. Clin Cancer Res 2013; 19: 1949-1959.

34. Di Bartolo BA, Cartland SP, Harith HH, et al. TRAIL-deficiency accelerates vascular calcification in atherosclerosis via modulation of RANKL. PLOS ONE 2013; 8: e74211.

35. Higashiyama M, Hokari R, Kurihara C, et al. Interferon-alpha increases monocyte migration via platelet-monocyte interaction inmurine intestinal microvessels. Clin Exp Immunol 2010; 162: 156-162.

36. Hansson GK. Immune mechanisms in atherosclerosis. Arterioscler Thromb Vasc Biol 2001; 21: 1876-1890.

37. Fernandes JL, Mamoni RL, Orford JL, et al. Increased Th1 activity in patients with coronary artery disease. Cytokine 2004; 26: 131-137.

38. Rho YH, Chung CP, Oeser A, et al. Novel cardiovascular risk factors in premature coronary atherosclerosis associated with systemic lupus erythematosus. J Rheum 2008; 35: 1789-1794.

39. Blanco-Colio LM, Martín-Ventura JL, Munoz-Garcia B, et al. TWEAK and Fn14: new players in the pathogenesis of atherosclerosis. Front Biosci 2007; 12: 3648-3655.

40. Schwartz N, Su L, Burkly LC, et al. Urinary TWEAK and the activity of lupus nephritis. J Autoimmun 2006; 27: 242-250. 
41. Chung C, Long A, Solus J, et al. Adipocytokines in systemic lupus erythematosus: relationship to inflammation, insulin resistance and coronary atherosclerosis. Lupus 2009; 18: 799 806.

42. Dong F, Zhang X, Ren J. Leptin regulates cardiomyocyte contractile function through endothelin-1 receptor-NADPH oxidase pathway. Hypertension 2006; 47: 222-229.

43. McMahon M, Skaggs BJ, Sahakian L, et al. Plasma leptin levels are associated with carotid artery plaque and intima-media thickness (IMT) in women with SLE and a matched population of healthy women. Arthritis Rheum 2007; 56 Suppl: S796.

44. McMahon M, Skaggs BJ, Sahakian L, et al. High plasma leptin levels confer increased risk of atherosclerosis in women with systemic lupus erythematosus, and are associated with inflammatory oxidised lipids. Ann Rheum Dis 2011; 70: 1619-1624.

45. Hahn BH, Lourencco EV, McMahon M, et al. Pro-inflammatory high-density lipoproteins and atherosclerosis are induced in lupus-prone mice by a high-fat diet and leptin. Lupus 2010 19: 913-917.

46. Michaelson J, Wisniacki N, Burkly L, Putterman C. Role of TWEAK in Lupus Nephritis: A bench-to-bedside review. J Autoimmun 2012; 39: 130-142.

47. Tiemessen MM, Jagger AL, Evans HG, et al. CD4+CD25+Foxp3+ regulatory $\mathrm{T}$ cells induce alternative activation of human monocytes/macrophages. Proc Natl Acad Sci USA 2007; 104: 19446-19451.

48. Reynolds HR, Buyon J, Kim M, et al. Association of plasma soluble E-selectin and adiponectin with carotid plaque in patients with systemic lupus erythematosus. Atherosclerosis 2010; 210: 569-574.

49. Blanco-Colio LM, Martín-Ventura JL, Munoz-Garcia B, et al. Identification of soluble tumor necrosis factor-like weak inducer of apoptosis (sTWEAK) as a possible biomarker of subclinical atherosclerosis. Arterioscler Thromb Vasc Biol 2007; 27: 916-922

50. Ouchi N, Kihara S, Arita Y, et al. Adiponectin, an adipocyte-derived plasma protein, inhibits endothelial NF-kappaB signaling through a cAMP-dependent pathway. Circulation 2000; 102: 1296-1301.

51. Aprahamian TR, Bonegio RG, Weitzner Z, et al. Peroxisome proliferator-activated receptor gamma agonists in the prevention and treatment of murine systemic lupus erythematosus. Immunology 2014; 142: 3633-3673.

52. Reilly MP, Lehrke M, Wolfe ML, et al. Resistin is an inflammatory marker of atherosclerosis in humans. Circulation 2005; 111: 932-939.

53. Baker JF, Morales M, Qatanani M, et al. Resistin levels in lupus and associations with disease-specific measures, insulin resistance, and coronary calcification. J Rheumatol 2011; 38: 2369-2375.

54. Ingelsson E, Larson MG, Fox CS, et al. Clinical correlates of circulating visfatin levels in a community-based sample. Diabetes Care 2007; 30: 1278-1280.

55. Brandt KJ, Kruithof EK, de Moerloose P. Receptors involved in cell activation by antiphospholipid antibodies. Thromb Res 2013; 132: 408-413.

56. Kiechl S, Lorenz E, Reindl M, et al. Toll-like receptor 4 polymorphisms and atherogenesis. N Engl J Med 347: 185-192.
57. Ameziane N, Beillat T, Verpillat P, et al. Association of the Tolllike receptor 4 gene Asp299Gly polymorphism with acute coronary events. Arterioscler Thromb Vasc Biol 2003; 23, e61-e64.

58. Wu XX, Guller S, Rand JH. Hydroxychloroquine reduces binding of antiphospholipid antibodies to syncytiotrophoblasts and restores annexin A5 expression. Am J Obstet Gynecol 2011; 205: 576.e7-576.e14.

59. Tam LS, Fan B, Li EK, et al. Patients with systemic lupus erythematosus show increased platelet activation and endothelial dysfunction induced by acute hyperhomocysteinemia. J Rheumatol 2003; 30: 1479-1484.

60. Duffau P, Seneschal J, Nicco C, et al. Platelet CD154 potentiates interferon-alpha secretion by plasmacytoid dendritic cells in systemic lupus erythematosus. Sci Transl Med 2010; 2: 47ra63. 